\title{
Spectroscopic Analysis of Single-Lined Spectroscopic Binaries with Unseen Companions
}

\author{
FAIG MUSAEV ${ }^{1}$, ILFAN BIKMAEV ${ }^{2}$, \\ and LAIMONS ZAČS ${ }^{3}$
}

${ }^{1}$ Special Astrophysical Observatory, Nizhnij Arkhyz, Russia

${ }^{2}$ Engelhardt Astronomical Observatory, Kazan State University Kazan, Russia

${ }^{3}$ Radioastrophysical Observatory, Riga, Latvia

Detailed abundance analyses have been carried out for 20 single-lined binaries with barium-star-like orbital elements using high-dispersion echelle spectra and the model atmosphere method. No substantial differences in the atmospheric abundances of 15 program stars relative to standard stars were found. Therefore, as a group, the single-lined spectroscopic binaries show atmospheric abundances similar to single stars, and the unseen companions did not have an influence on the atmospheric abundances of the primary stars studied.

The full version of this report has appeared in $A \& A$ Supp., 122, 31, 1997 as a paper on "An abundance analysis of the single-lined spectroscopic binaries with barium stars-like orbital elements. I. Analysis and results" by L. Začs, F. A. Musaev, I. F. Bikmaev, and O. Alksnis. 